\title{
Enseñabilidad. \\ La necesidad de una revisión crítica en contabilidad
}

Recibido: 15 de marzo de 2013

Aprobado: 15 de junio de 2013

Yuliana Gómez Zapata ${ }^{1}$

Gómez, Y. (2013). Enseñabilidad. La necesidad de una revisión crítica en contabilidad. Activos 21, 65-86.

Clasificación JEL: Z 19

\section{Resumen}

Este trabajo tiene como preocupación principal la discusión conceptual respecto de la enseñabilidad en contabilidad desde una revisión crítica, es decir, la necesidad de comprender las formas particulares sobre las cuales se enseña en contabilidad, de tal forma que se contribuya al mejoramiento de las prácticas educativas contables. La metodología procesal que se plantea refiere una provocación al pensamiento crítico; una discusión conceptual respecto a la enseñabilidad, vista a través de la contabilidad. Una propuesta praxiológica desde la alteridad que intenta develar la importancia de una contabilidad enseñable y comprensible, además de ética y humana. Los resultados de la investigación se sintetizan en la obligación de comprender las formas sobre las cuales se enseña en los programas de Contaduría Pública en Colombia, se explicita la relevancia que tiene la vinculación sociohumanística con la contabilidad como entronque potenciador de

1 Contadora Pública. Magister en Ciencias Contables y estudiante de Sociología de la Universidad de Antioquia. Docente tiempo completo adscrita al programa de Contaduría Pública del Tecnológico de Antioquia, Medellín - Colombia. Correo electrónico: ygomezza@tdea.edu.co 
la formación integral de sujetos. Por último, se plantean alternativas de enseñabilidad que enfocan la comprensión del quehacer contable en la solución de la problemática social.

\title{
Palabras clave
}

Educación contable, enseñabilidad, prácticas de enseñanza-aprendizaje, contabilidad.

\section{Gómez, Y. (2013). Teachability. The need for a critical review in accounting. Activos 21, 65-86.}

\begin{abstract}
This paper has as main concern the conceptual discussion regarding the teachability in accounting from a critical review, i.e., the need to understand the particular forms on which accounting is taught, so that it contributes to the improvement of educational accounting practices. The procedural methodology presented refers a provocation to critical thinking; a conceptual discussion regarding teachability, seen through accounting. A praxeological proposal from the otherness that intends to reveal the importance of a teachable and understandable accounting, in addition to ethical and human. The research results are summarized in the obligation to understand the ways in which is taught in the Public Accounting programs in Colombia, it makes explicit the relevance of social and humanistic relation with accounting as an enhancing connection in the comprehensive formation of individuals. Finally, different teachability alternatives are presented which focus the understating of the accounting tasks in the solution of social problems.
\end{abstract}

\section{Keywords}

Accounting education, teachability, teaching-learning practices, accounting. 
Gómez, Y. (2013). Enseignement. La nécessite d'une révision critique en comptabilité. Activos 21, 65-86.

\section{Résumé}

La préoccupation principale de cette étude, est la discussion conceptuelle au sujet de l'enseignement de la comptabilité depuis une révision critique, c'est à dire, la nécessité de comprendre les formes particulières sous lesquelles la comptabilité est enseignée, de façon telle à contribuer à l'amélioration des pratiques éducatives comptables. La méthodologie procédurale formulée, évoque une provocation de la pensée critique ; une discussion conceptuelle au sujet de l'enseignement, vue à travers la comptabilité. Une proposition praxéologique depuis l'altérité, qui tente de révéler l'importance d'une comptabilité qui puisse être enseignée et comprise, et en outre soit humaine et éthique. Les résultats de la recherche se synthétisent dans l'obligation de comprendre les formes d'enseignement des programmes d'expertise comptable en Colombie, la pertinence de la liaison socio-humanistique avec la comptabilité se précise, en tant que lien potentialisateur de la formation intégrale de sujets. Finalement, des alternatives d'enseignement qui abordent la compréhension de la tâche comptable dans la solution de la problématique sociale, sont soulevées.

\section{Mots clés:}

Education comptable, enseignement, pratiques d'enseignement- apprentissage, comptabilité. 
Enseñar no es transferir conocimiento, sino crear las posibilidades para su propia producción o construcción... Jamás acepté que la práctica educativa debería limitarse sólo a la lectura de la palabra, a la lectura del texto, sino que debería incluir la lectura del contexto, la lectura del mundo.

Freire. (2000)

\section{Prolegómenos: provocación al pensamiento crítico}

La comunidad académica contable delinea, desde hace algunos años, caminos sobre los cuales la disciplina pueda construir modelos materiales y mentales que le permitan aprehender las diferentes problemáticas de la sociedad particularizada y darles posibles soluciones ${ }^{2}$. Es complejo para académicos, estudiantes y directivos de los programas de Contaduría Pública del país, encausar discusiones epistemológicas desde la contabilidad, teniendo en cuenta el alto grado de tecnicidad e instrumentalización ${ }^{3}$ que comporta la estructura de los programas de Contaduría Pública, y el peso que ello tiene en el imaginario colectivo de los actores contables.

De igual manera, es importante resaltar la labor comprometida que tienen algunos académicos contables ${ }^{4}$ en pro de la relevancia epistemológica

2 Revisar en contexto la obra del profesor argentino Carlos Luis García-Casella, a partir del cual se podría ampliar la referencia y posibilitar la discusión académica.

3 Desde las reflexiones que plantea el profesor William Rojas (revisar textos consultados en los referentes bibliográficos), entre otros, se podría soportar la relevancia que tiene para los contables la vigencia del pensamiento técnico e instrumental en la formación y el ejercicio profesional.

4 Para mayor comprensión revisar la obra de los profesores William Rojas Rojas, Edgar Gracia López y Guillermo León Martínez Pino, en las cuales se hace una interesante descripción de lo planteado, puesto que la racionalidad técnicoinstrumental ha ganado fuerza en las discusiones académicas que se interesan por formar estudiantes de Contaduría Pública, y ha convocado esfuerzos importantes 
y cognoscitiva de la contabilidad, a partir de su vinculación ineludible con la resolución de problemas sociales y empresariales, lo cual potencia la seriedad del ejercicio profesional contable como generador de confianza pública.

De la misma forma, se han evidenciado falencias en cuanto a los métodos de enseñanza que dentro de las universidades se aplican, ya que la contabilidad históricamente ha sido concebida más como una técnica que como una disciplina científica que contiene un cuerpo cognitivo, lo cual ha propiciado unas metodologías de enseñanza-aprendizaje poco interdisciplinarias y poco transformadoras de la realidad (Gómez, 2012, p. 4).

El ejercicio que constituye pensar y repensar la contabilidad y la realidad que representa, es una acción comprometida que debe ejercerse desde la academia y como sujetos insertos en la realidad social con un alto sentido ético y estético que permite comprender las necesidades de la sociedad y de las organizaciones que la componen, en directa relación con la contabilidad y su aporte a la solución de los problemas fundamentales.

Ahora bien, el ejercicio de pensar y repensar la contabilidad, sus prácticas de enseñanza-aprendizaje; los modelos a partir de los cuales aprehende la realidad social y organizacional; y los sujetos que la ejercen, entre otros asuntos relevantes del quehacer contable, no es suficiente para generar transformaciones concretas o estructurales, puesto que las apuestas que se develan en el imaginario colectivo de los actores contables se sustentan en los mismos postulados, paradigmas y principios, utilizando metodologías de enseñanza poco renovadoras y con un alto grado de monodisciplinariedad.

A partir de lo anterior, cabe la posibilidad de seguirnos preguntando -como docentes- qué, cómo y para qué enseñar en contabilidad, debido a que las teorías ortodoxas de la educación, las mismas que se introducen en

para ampliar dichos imaginarios y hacer un poco más holístico el accionar de la contabilidad. 
los discursos de la educación contable universitaria, reducen la posibilidad de generar alternativas de enseñanza que abran los espectros de actuación contable, y que a su vez, diversifiquen el acercamiento de los estudiantes de Contaduría Pública hacia otros campos de la vida social, más allá de la preocupación constante sobre el devenir empresarial.

Por su lado, las nuevas teorías y perspectivas críticas de la educación (la Escuela Nueva, la educación antiautoritaria, la perspectiva comunicativa, la escuela y los movimientos sociales alternativos, la paralización de la acción, entre otras) que parten desde varios paradigmas científicos adscritos a diferentes posturas ideológicas, políticas y culturales, ayudan a comprender los contextos contemporáneos del mundo, de las disciplinas que lo interpretan y de los comportamientos de los sujetos que lo habitan.

Enseñar en contabilidad desde las teorías, modelos y metodologías ortodoxas, constituye una acción reducida desde el ejercicio pedagógico, puesto que los nuevos contextos exigen mayor comprensión de los roles que se ejercen en el desarrollo de la acción, es decir, se requiere conocer profundamente la necesidad de la acción de enseñar, el sentido y las condiciones requeridas para tal ejercicio, y por supuesto, cuáles son las realidades que se mejoran a partir de la enseñanza.

La enseñabilidad, tal y como lo plantea Flórez (1999, p. 34):

Es una característica derivada del estatuto epistemológico de cada ciencia o disciplina referida a sus rasgos de racionalidad y de sintaxis, de contenido teórico y experiencial, que distingue el abordaje de sus problemas y condiciona específicamente la manera como cada disciplina puede o debe enseñarse.

Es por esto que se requiere que cada vez más, los profesores insertos en la enseñanza de la contabilidad desde los diferentes claustros universitarios, visualicen prácticas de enseñanza que verdaderamente sean enseñables desde la contabilidad, teniendo como punto de partida los contextos 
universitarios y organizacionales, de tal manera que se planteen alternativas que mejoren dichas prácticas en pro de la formación integral y de excelencia de los futuros profesionales contables.

Con base entonces en la discusión que se ha venido suscitando en este escrito, se explicita que, derivada de un proceso de investigación cualitativo, tiene como preocupación principal, la discusión teórica y conceptual respecto de la enseñabilidad de la contabilidad desde una revisión crítica, es decir, la necesidad de comprender las formas particulares sobre las cuales se enseña en contabilidad, de tal forma que se contribuya al mejoramiento de las prácticas educativas contables.

La ponencia recorre una metodología procesal, que refiere una provocación al pensamiento crítico, una discusión conceptual respecto a la enseñabilidad, y particularmente vista desde la contabilidad, una propuesta praxiológica desde la alteridad que intenta develar la importancia de una contabilidad enseñable y comprensible, además de ética y humana. Por último, se concretizan los resultados de la reflexión investigativa en la necesidad de comprender las formas sobre las cuales se enseña en contabilidad, teniendo en cuenta las aristas de su estatuto epistemológico, su racionalidad, sus constructos teóricos y experienciales, de tal forma que pueda concretarse el concepto crítico en el desarrollo efectivo de las prácticas de enseñanza.

Del mismo modo, explicita la relevancia que tiene la vinculación sociohumanística con la contabilidad como entronque potenciador de la formación integral de sujetos, además de plantear alternativas de enseñabilidad en contabilidad, tales como la construcción de comunidades investigativas, la inter y la transdisciplinariedad, la investigación formativa, las metodologías activas, entre otras, las cuales enfocan la comprensión del quehacer contable en la solución de la problemática social. 


\title{
La enseñabilidad: Elementos críticos para una fundamentación conceptual
}

\author{
"Enseñar no es transferir conocimiento, \\ sino crear las posibilidades para su propia \\ producción o construcción".
}

Freire. (2000)

Las reflexiones contenidas en este documento, tal y como se ha indicado, reflejan el trabajo investigativo que se circunscribe en la línea de investigación en educación contable, puesto que la preocupación frente a los procesos de enseñanza-aprendizaje como educacionales, debe constituirse en acción obligada de estudiantes, profesores y directivos de los diferentes claustros universitarios en los cuales se imparte la Contaduría Pública como profesión liberal.

Este apartado pretende avanzar en las formas conceptuales que se discurren del ejercicio investigativo en términos de la educación y formación contable, de tal manera que se revisen los fundamentos epistemológicos y teóricos que soportan dicha discusión conceptual; además de tener en cuenta que los fundamentos revisados se corresponden con la necesidad de fundamentar desde unas corrientes de pensamiento más amplias, holísticas y menos tradicionales, las problemáticas que devienen del quehacer educativo contable.

En sentido contrario, y aunque no se ha precisado la reflexión desde las teorías tradicionales de la educación, es necesario tener presente las reflexiones que se tejen en esos sentidos, incluso desde las posturas más ortodoxas de la sociología de la educación, como lo es la visión de Émile Durkheim, quien

(...) plantea cuestionamientos sociológicos en términos de qué enseñar, cómo enseñar y para qué enseñar, además de entender que la educación es ante todo un hecho social, el cual, desde una sociedad determinada, 
moldea al sujeto en una acción individualizada y colectivizada. (Gómez, 2012, p. 14).

Cuando la preocupación por las formas de enseñar y educar en contextos universitarios, responde a cuestionamientos más profundos, mucho más amplios, críticos y comprensivos, los planteamientos de Durkheim (2000) pueden quedarse cortos para su interpretación, es decir, se requieren elementos de análisis crítico que permitan auscultar a fondo los presupuestos epistemológicos y contextuales que subyacen en la acción de enseñar y aprender.

Ahora bien, cuando se plantea la discusión desde el punto de vista de la enseñanza, se debe significar que dicha acción puede ser desarrollada verticalmente de generación a generación, ${ }^{5}$ pero existen otras miradas que contemplan la posibilidad de discusión desde puntos críticos de inflexión, como los planteados por Gallego y Pérez (1998, p. 25), cuando señalan que:

La actividad de enseñar es afectada por las concepciones de aprendizaje, de alumno y alumna, de profesor y profesora, por las intencionalidades curriculares y por los compromisos epistemológicos mismos de los profesores. De hecho, lo es también por la clase de formación profesional de que han sido objeto los profesores y profesoras, con el fin de asumir sus compromisos, ya sea como operarios o como trabajadores de los saberes en sus dimensiones y problemas epistemológicos, pedagógicos y didácticos.

La enseñanza como proceso importante en la formación de sujetos, puede quedarse un tanto corta, en la medida en que los sujetos que ejercen la acción desconocen el sentido de ejercerla, además de las posibilidades que se le genera al sujeto que recibe la acción, en términos de su subjetividad y su estructuralidad. En ese sentido, se convoca a la apertura del pensamiento

5 Ampliar el concepto en el texto de Émile Durkheim Educación y Sociología, en el cual se plantean aspectos relevantes para el análisis conceptual de este acápite. 
desde las teorías heterodoxas de la educación, ${ }^{6}$ aquellas que revierten los elementos tradicionales de la formación, a partir de los cuales los sujetos se forman pero desconocer la esencia y el objeto de esa formación. En este sentido, el concepto de enseñar debe trascender, es decir, replantear su misión y generar posibilidades de cambio estructural desde el punto de vista de la enseñabilidad. ${ }^{7}$

Para ejemplarizar un poco más el concepto, Flórez (1999, p. 35) aporta los siguientes análisis desde un punto de vista crítico:

La enseñabilidad es una característica derivada del estatuto epistemológico de cada ciencia o disciplina referida a sus rasgos de racionalidad y de sintaxis, de contenido teórico y experiencial, que distingue el abordaje de sus problemas y condiciona específicamente la manera como cada disciplina puede o debe enseñarse.

"La enseñabilidad responde a la pregunta ¿por qué las matemáticas requieren enseñarse de manera diferente a la historia?” (1999, p. 34).

Igualmente, desde las reflexiones que han planteado los docentes de la Universidad Pontificia Bolivariana (Medellín), López (2004) plantea que a partir de la definición expuesta por Flórez se puede inferir que:

(...) aunque la pregunta por la enseñabilidad es realizada por la pedagogía, está por sí misma no puede dar cuenta de la respuesta, pues en un primer momento el concepto se despliega a partir de una mirada interna de la ciencia cuando se indaga a sí misma, a cerca de sus condiciones de objetividad; su racionalidad; sintaxis y contenido teórico y experimental. (...)

6 Véase el texto Nuevas perspectivas críticas en educación, en el cual se precisan elementos importantes expuestos por los autores Paulo Freire, Orlando Fals Borda, Ramón Flecha, Henry Giroux, Manuel Castells (1994), entre otros, de tal forma que se amplía la importancia que revisten tales en este documento.

7 En palabras de los profesores Gallego y Pérez (s. f.), este concepto precisa que los profesores y profesoras se deben comprometer con un proyecto epistemológico, pedagógico y didáctico de carácter investigativo y teóricamente fundamentado. En tal sentido se es coherente con la intencionalidad del concepto. 
es necesario tejer una serie de relaciones que se inscriben en el marco de referencialidad educativo que modifica la mirada interna del ser. (...) la reflexión acerca del marco de referencialidad educativo es un asunto que compete (...) al profesor, quien a partir de su mirada pedagógica descubre y hace explícitas las relaciones entre la enseñabilidad y las condiciones del aprendiz (educabilidad) para acceder a las ciencias, las disciplinas y los saberes, y a partir de allí formar su pensamiento y construir su propia comprensión acerca de la lógica y sintaxis del saber. (2004, p. 139).

Es importante reconocer en este punto de la discusión, que si bien desde la contabilidad apenas se comienzan a precisar elementos de tipo conceptual y epistemológico que se insertan en la discusión de la enseñabilidad, vista desde la enseñanza de la contabilidad como disciplina social y aplicada, ${ }^{8}$ existen docentes contadores que se han interesado por la problemática de la educación contable, tal y como se expresó en la fase introductoria de este escrito, lo cual valida la relevancia de la postura reflexiva frente a la educación y formación de profesionales contables.

En directa relación, el trabajo de investigación magisterial "Enseñabilidad y educabilidad de la contabilidad: una discusión desde las teorías críticas", presentada por la autora de este texto, introduce en el discurso la necesidad de comenzar a hablar en tales términos, puesto que las teorías ortodoxas de la educación, restringen las formas de pensar, de actuar y de ver el mundo, aduciendo a prácticas conservadoras y poco emancipadoras.

En tal sentido se plantea que:

La enseñabilidad es entonces, un concepto que requiere el desarrollo de una enseñanza intencional a partir de las características y necesidades que tenga cada disciplina, desde el punto de vista epistemológico o gnoseológico, que debe tener en cuenta varios aspectos como a quién se le enseña, para qué

8 Según Sarmiento, la contabilidad es “(...) una disciplina social, fáctica y aplicada, que estudia el control de la riqueza, a través de sistemas de información referidos a los procesos de medición, valoración y control de los recursos materiales, sociales y naturales (...)"(2007, p. 55). 
y por qué ha de enseñarse, qué condiciones se requieren para pensar las posibilidades de enseñanza, y qué realidades transforma aquello que es enseñable, puesto que la enseñabilidad, como proceso retroalimentado, tiene en cuenta la cultura, la historia, lo económico, lo social y las condiciones de los sujetos que intervienen en ese proceso. (Gómez, 2012, p. 17).

Ahora bien, tal caracterización contempla la necesidad inminente de detallar las condiciones a partir de las cuales se pretende ejercer el proceso de la enseñabilidad, y más precisamente desde la disciplina contable, a lo cual Flórez (1999), vuelve a presentar su propuesta en términos de lo pedagógico cuando aduce que dichas características se constituyen en la comunicación entre los sujetos que hacen parte del ejercicio disciplinal, las características del rigor disciplinal, su racionalidad, su lógica interna, sus grados y niveles de epistemologización, su lenguaje empírico, y todos aquellos condicionantes que se ejercen sobre las disciplinas concretas que desean ser enseñables. Ahora bien, es importante que los docentes que intervienen en el proceso, dominen la ciencia o disciplina que pretenden enseñar, de lo contrario, el proceso puede convertirse en una distorsión del proceso de enseñanza-aprendizaje.

Para concretar un poco el asunto en discusión, Flórez (1999, p. 37) concluye:

Lo más importante de la enseñabilidad como una propiedad derivada del estatuto epistemológico de cada disciplina es que sus rasgos de racionalidad, comunicabilidad y orden propios permiten configurar una pauta orientadora, una señal "ejemplar" que le suministra al pedagogo un punto de partida y un apoyo disciplinar específico para romper con sus recetas, métodos y diseños generales y abstractos de la didáctica general convencional, y disponerlo a diseñar su enseñanza de manera específica, teniendo en cuenta las particularidades de cada temática disciplinar y sus repercusiones para el aprendizaje de alumnos concretos.

La enseñabilidad es entonces, desde las reflexiones que se han contemplado en este texto, pero además de las muchas que pueden derivarse de las 
discusiones académicas de pedagogos que han trabajado suficientemente dicho concepto, una apuesta ineludible de docentes y directivos de los programas académicos universitarios, por mejorar las prácticas educativas utilizadas para la formación específica de los profesionales.

Pero además, si esta discusión se discurre en las prácticas de enseñanza, pedagógicas, didácticas, educacionales y formativas esgrimidas desde la contabilidad, se estaría avanzando considerablemente en el reconocimiento del estatuto epistemológico de la contabilidad, en la trascendencia de la referenciación empírica hacia el reconocimiento de metarrelatos como metodología de construcción disciplinal, en la utilización de elementos taxativos que contextualicen la disciplina contable y esta sea enseñable y no enseñada.

\section{Alternativas de enseñabilidad: Propuesta praxiológica desde la alteridad}

La educación es fundamental para la toma de conciencia, la formación y el crecimiento de la sociedad (...) Tenemos que cambiar el pensamiento cartesiano por uno holístico e integrador.

Adolfo Pérez Esquivel

Como se ha planteado, la contabilidad -al igual que otras disciplinasrequieren de elementos problematizadores que ayuden a comprender los contextos y las dinámicas dialécticas de la sociedad, la ciencia y por su puesto los sujetos que la componen. Este asunto particular no puede aislarse de las discusiones que se enmarcan en el campo de la educación y la formación de profesionales de la Contaduría Pública, puesto que la forma a partir de la cual se enseña, se educa y se forma estudiantes de Contaduría Pública, repercute directamente en su compromiso ético y social en ejercicio de su profesión. 
En este sentido, plantear alternativas para hacer de la enseñanza de la contabilidad algo mucho más holístico, contextuado y dinámico, requiere de un compromiso mayor de quienes se piensan constantemente la disciplina, en el entendido de que tal acción, permite auscultar las prácticas dentro y fuera del aula, de tal forma que la contabilidad se convierta en un saber enseñable que se preocupe por construir sujetos con sentido crítico, capaces de pensar y repensar la sociedad en la que viven con el propósito de ayudar a su constante transformación.

La caracterización de alternativas de enseñabilidad que permitan enseñar la contabilidad de una forma diferente a las matemáticas, a la economía, a la estadística, etc., a partir de una revisión consciente de su estatuto epistemológico, teórico-conceptual y experiencial, ratifica la necesidad de distinguir la forma más pertinente para ser enseñada. En este sentido, al hacer una contrastación respecto al particular, se encuentra algunas de las prácticas utilizadas en la enseñanza de otras disciplinas que han avanzado en tal discusión académica, ${ }^{9}$ puesto que se pueden transferir tales experiencias a la contabilidad.

Para ejemplarizar un poco la discusión suscitada, se delimitan algunas de las alternativas de enseñabilidad, comentadas en este documento, en su recorrido procesal, de tal manera que se introduzcan elementos de análisis que permitan comprender el alcance que tienen para la enseñanza de la contabilidad, desde los claustros universitarios y por fuera de ellos; apoyada en las estructuras curriculares, en la formación de sujetos conscientes y éticos.

9 Es importante para este punto, consultar la experiencia de la Universidad de Antioquia, la Universidad Pedagógica Nacional y la Universidad Pontificia Bolivariana en Colombia y las Universidades Central y Experimental Simón Bolívar en Venezuela, además de incluir en tal cuestión, a la Universidad de la Habana Cuba, quienes, además de otras en el mundo, han comprendido la necesidad inminente de demarcar las prácticas de enseñanza-aprendizaje y las alternativas para hacer enseñable la ciencia desde su particularidad. 


\section{Intercambio de conocimientos profesor-estudiante- profesor: La construcción de comunidades investigativas y académicas}

Quienes enseñan la contabilidad como disciplina científica ${ }^{10}$ deben comprender que el rol privilegiado del docente, trae consigo un sinnúmero de connotaciones, las cuales permiten una interlocución constante entre el estudiante y el docente, de tal forma que se pueda construir conocimiento horizontal y sin la concepción durkheimiana (2000, p. 12) mezquina de que "quien sabe es el profesor, y el alumno solo puede aprender lo que este enseña de forma vertical y sin reparaciones".

La necesidad de auscultar alternativas que permitan una verdadera enseñabilidad de las disciplinas sociales y de la contabilidad particularmente, hace apremiante la discusión, y ponen en consideración de los lectores que, este tipo de prácticas, que se pueden dar de forma simultánea desde el aula hasta los espacios externos a ella, son esenciales para la formación de sujetos conscientes y capaces de responder ante situaciones complejas de la vida social donde la contabilidad como portadora de conocimientos claves para el desarrollo de la misma, debe aportar a la concreción de escenarios donde se pueda dar dicha relación.

Lo que se quiere plantear entonces, es que los docentes, actores principales en el proceso, comprendan desde una perspectiva crítico-reflexiva, que es necesario plantear con los estudiantes una relación de pares, en el entendido de que en el proceso de enseñanza-aprendizaje el estudiante también aporta conocimientos nuevos al docente que pueden enriquecer el acto formativo.

En este punto de la discusión, el hecho de que los docentes reconozcan el valioso aporte de los estudiantes como actores fundamentales en el

10 Es necesario comprender que la contabilidad debe enseñarse como disciplina científica, puesto que de no hacerlo, se corre el riesgo de proceder inocuamente en términos de las alternativas propuestas. 
proceso de enseñanza-aprendizaje, no significa que estos últimos asuman una actitud del "todo vale", donde no es necesaria la lectura, la discusión y la argumentación frente a las distintas dinámicas académicas particularizadas en sus objetos de estudio, sino que su opinión es suficiente para formarse como profesional y como sujeto, pues ello constituye un error formativo en la medida en que los docentes no hagan saber a los estudiantes que deben comprometerse con su rol específico, y no conformarse con el facilismo académico propio de las generaciones contemporáneas.

Ahora bien, la enseñanza de la contabilidad debe desmitificar sus formas técnicas e inocuas de transferir conocimientos verticalmente desde la memorística y el pragmatismo, pues de esa forma, los estudiantes no se convierten en sujetos pensantes, sino en tecnócratas al servicio de una sociedad desigual e injusta, propia de la modernidad.

\section{Desde la inter hacia la transdisciplinariedad}

En este punto, no se hace necesario discernir sobre la importancia de la inter y de la transdisciplinariedad de las ciencias; en la misma vía, aclarar que la transdisciplinariedad desde el currículo es fundamental para la comprensión crítica de la realidad y para el aporte de una mejor enseñanza de la contabilidad, puesto que brinda elementos para resolver los problemas de la sociedad desde la contabilidad y con el aporte de otras disciplinas, lo cual hace que el estudiante comprenda que esas otras disciplinas no son rellenos en su formación, sino que son contenidos necesarios para la comprensión y transformación de la realidad.

Igualmente se hace urgente la vinculación interdisciplinaria en los currículos de Contaduría Pública, pues consecuentemente con la preocupación que aborda esta ponencia, la enseñabilidad de la contabilidad depende en gran medida de las posibilidades de relacionamiento con otros saberes y la correcta distinción de los aportes que dichos saberes hacen a la contabilidad, pues esta última debe enseñarse bajo sus particularidades y no desde las necesidades y lógicas de las demás disciplinas afines o cercanas a ella. 
Por lo anterior, resulta imperioso que se introduzca en el discurso contable, tanto desde el punto de vista de la educación, como de la formación de sujetos, la importancia de la interdisciplinariedad como posibilitadora de la construcción de discurso teórico y disciplinar desde una perspectiva crítica.

\section{La investigación formativa}

La universidad plantea la necesidad de hacer docencia, investigación y extensión, como pilares fundamentales de su misión; esas prácticas se han institucionalizado de tal forma que se hace difícil que los estudiantes entren en su dinámica a partir de su rigurosidad funcional, y la desvinculación que ejercen los docentes en el proceso, al creer que los estudiantes no han alcanzado el rigor necesario para entrar en esas lógicas investigativas.

Lo que se plantea entonces, es que se propicien espacios dentro del aula, que le permitan al estudiante hacer parte de los procesos investigativos desde lo simple, de tal forma que este sea capaz a de crear y recrear, de manera autónoma, asuntos de la vida social aportantes para el desarrollo de la disciplina y de los sujetos que la estudian. A este proceso se le conoce como investigación formativa y su aplicación en contabilidad es absolutamente necesaria porque genera la interacción crítica de los estudiantes con el mundo exterior y desde cada uno de los núcleos problematizadores de su objeto de estudio particular.

(...) el tema de docencia-investigación es clave para la teoría crítica. No podemos plantear en las aulas universitarias que la docencia va por un lado y la investigación por otro; esos procesos están entrelazados. El estudiante es un investigador permanente, todos los días está descubriendo, cada instante está reflexionando, es un investigador, es decir, yo no concibo las universidades sin investigación, no concibo el proceso de enseñanzaaprendizaje sin investigación. Es importante que el estudiante incluso se inscriba en ese proceso de lo que llamamos investigación formativa en el aula de clase, que él mismo empiece a descubrir, que ahí mismo empiece a pensar; ese también es un aporte de la teoría crítica. (Zapata, 2011). 
La investigación formativa como alternativa de enseñabilidad, aunque podría entenderse igualmente como una práctica educable, es referida por profesores universitarios ${ }^{11}$ quienes la defienden como una de las alternativas más pertinentes para el trabajo de enseñanza-aprendizaje.

\section{Diagnósticos de reconocimiento de la realidad social como aporte a la discusión y al debate}

La contabilidad, a través de los diferentes núcleos de estudio enseñados en la universidad, debe propender por hacer un reconocimiento de los contextos en los cuales se desenvuelve, de tal forma que los estudiantes no se arriesguen, desde el desconocimiento, a reconocer una realidad que les es distante y ajena. La aplicación de esta alternativa, debe ir de la mano de la didáctica, puesto que no es posible reconocer el espacio y el contexto a partir de la mera narración magistral, sino que se requiere que los docentes planteen alternativas didácticas que le permitan al estudiante introducirse en el campo e indagar por sus cuestionamientos fundamentales, de tal forma que se pueda hacer una correcta relación entre lo que se estudia y la realidad que se desea intervenir.

Lo anterior, puede atribuirse a una visión más humana y dialéctica del mundo; $y$, es urgente comprender que son la universidad y las disciplinas que la componen, quienes deben hacer diagnósticos situacionales, contextualizarse y brindar soluciones a las problemáticas que las atañen. Esto es plausible en contabilidad.

Es probable que este tipo de prácticas se estén desarrollando en diferentes universidades y programas de Contaduría del mundo entero, y por tanto, es responsable precisar que se está haciendo una referenciación en

11 En audio y texto digital, se tienen evidencias de entrevistas a diferentes profesores, tanto contadores como educadores y sociólogos, que se han enfocado hacia la problematización de las disciplinas concretas en cuanto a su forma de enseñanza; en tales términos estos apuntan a la relevancia que tiene la investigación formativa para la formación de profesionales y personas. 
términos de algunas experiencias de docencia selectivas y en función de la literatura contable nacional y latinoamericana que enfocada en la problemática educativa.

\section{El uso de metodologías activas para la construcción de reflexividad e interés}

Reconocer que a lo largo de la historia las prácticas pedagógicas se han enfocado principalmente en la pedagogía tradicional; aquellas que han delineado la formación de sujetos, quienes a su vez se han convertido en seres a-críticos, repetitivos y memorísticos. Las prácticas pedagógicas tradicionales, se han enfocado básicamente en el saber del docente, es decir, lo que este transmite al estudiante a través del proceso de enseñanza, dejando de lado elementos sumamente valiosos que componen el proceso de enseñanza-aprendizaje desde el punto de vista de la enseñabilidad.

En este orden de ideas, se proponen en la actualidad nuevos enfoques desde otras visiones del mundo, aquellos que pueden devenir de las pedagogías activas, la pedagogía desarrolladora, los procesos conscientes y constructivos, que centran la atención en el desarrollo cognitivo del individuo y potencian sus capacidades para el aprendizaje.

El pensamiento crítico permite entonces que el individuo situado en contexto, sea consciente de su proceso de aprendizaje y proyecte sus conocimientos hacia la transformación social. Tal y como lo expresa la profesora Ángela Urrego, "posibilitar que los objetos de enseñanza puedan ser vivenciados de acuerdo con el contexto y hacer uso de metodologías activas que acerquen a los estudiantes al descubrimiento para ofrecer soluciones a problemas", ${ }^{12}$ es una de las alternativas que la contabilidad debe explorar para que su saber sea enseñable de forma acorde con las necesidades del contexto, puesto que de otra manera, se estaría presenciando la disparidad

12 Entrevista concedida a la autora de este texto, el 11de febrero de 2012 en Medellín. Registro documentado en texto digital. 
de enseñar desde otros saberes el contable, y propiciando de forma errática la irreflexividad y el desinterés.

\section{Consideraciones finales}

Conocer las discusiones que actualmente existen en las comunidades académicas - particularmente en contabilidad- permite pensar en otras posibilidades de relacionamiento, de puntos de vista, de imágenes, de formas, de relatos, es decir, otras posibilidades de ver el mundo, ya no desde lo siempre ortodoxo y confesional, sino desde lo alterno, lo heterodoxo.

La contabilidad como disciplina social no debe estar sometida únicamente a la racionalidad técnico-instrumental, como lo señala Horkheimer (2002), como única forma de ver el mundo y explicarlo, puesto que lo contable puede estar mucho más comprometido con los devenires del mundo y de sus contextos. En tal sentido, la formación no debe convertirse en un cliché, a partir del cual la mayoría de los programas de Contaduría Pública forman profesionales íntegros e integrales, pero realmente no conocen lo que ello significa.

Formar desde lo cognoscitivo, al igual que desde lo instrumental y lo humano es requerido para formar sujetos integrantes de un mundo complejo y dinámico. No es correcto preponderar la formación técnica cuando se estructura un perfil profesional que corresponde a la situación de otros elementos igualmente importantes. El discurso de la formación integradora no puede ser un modismo más de las políticas institucionales que requieren buenos tecnócratas pero pésimos críticos.

Las alternativas de enseñabilidad que se han planteado a lo largo de este documento ponen de manifiesto el compromiso -aún posible- de contribuir al mejoramiento de las prácticas educativas y de enseñanza-aprendizaje en contabilidad, las cuales han estado plagadas de un alto grado de ortodoxia en las concepciones de teóricos, docentes y estudiantes. 
Las prácticas educativas y formativas, entendidas como alternativas de enseñabilidad -sin trivializar el concepto-, que se deberían tener en cuenta para la enseñanza de la contabilidad en los espacios universitarios institucionales y desinstitucionalizados, son aquellas que se apoyan teóricamente en la crítica y la comprensión para transmitir conocimiento y para comprender el mundo, mejorarlo y humanizarlo.

Por lo anterior, los administradores de los programas académicos de Contaduría Pública en Colombia deben reflexionar y comprender su papel histórico en la construcción de sujetos y de sociedad, puesto que no se puede seguir concibiendo a la contabilidad como una práctica aséptica, técnica e inclusive artística socialmente reconocida, en la cual sus actores fundamentales, no son más que simples tecnócratas al servicio de los intereses de la empresa, como único núcleo de desarrollo contable. 


\section{Referencias bibliográficas}

Castells, M. (1994). Nuevas perspectivas críticas en educación. Barcelona: Paidós. Durkheim, E. (2000). Educación y sociología. (2ª ed.) Barcelona: Península.

Flórez, R. (1999). Enseñabilidad y pedagogía. Acción Pedagógica, 8 (01), 34-37.

Freire, P. (2000). Pedagogía del oprimido. México: Siglo XXI.

Gallego, R. y Pérez, R. (S.F.). Aprendibilidad, enseñabilidad y educabilidad: una discusión. Recuperado de http://www.pedagogica.edu.co/storage/rce/articulos/rce36-37_07vida.pdf.

Gómez, Y. (2012). Enseñabilidad y educabilidad de la contabilidad: primer acercamiento. Recuperado de http://www.alafec.unam.mx/memoria_xiii.php.

Horkheimer, M. (2002). Crítica de la razón instrumental. Madrid: Trotta.

López, B. (2004). Acerca de la enseñabilidad. Revista Textos, 08, 134-148.

Sarmiento, H. (2007). Retorno a la idea. Elementos metodológicos para la construcción de conceptos propedéuticos de investigación contable. Lúmina, 08, 43-57.

Zapata, G. (2011). Entrevista concedida a la autora. Medellín. Registro documentado en audio y texto digital. 